\title{
ROTATIONAL SPEED LIMIT OF ACTIVELY CONTROLLED JOURNAL BEARINGS AT STABLE LUBRICATION
}

\author{
J. Tůma ${ }^{*}$, A. Víteček ${ }^{* *}$
}

\begin{abstract}
The paper deals with the stability of the journal motion in hydrodynamic journal bearings while actively controlling their vibrations by the position of the bearing bushing. The bearing journal motion can only be stable when lubrication is stable. The first chapter of the paper contains a description of the conditions for forming a stable oil film. The paper deals with the mathematical model of the journal bearing with a fixed and movable bushing. The analysis uses verified mathematical models in the form of motion equations describing the motion of the journal axis in the plane which is perpendicular to the rotor axis in contrast to the magnetic bearing; the equations are coupled. The stability analysis aims to determine the critical rotational speed of the rotor at which vibrations of the whirl type are generated. The stability analysis tool is the Nyquist Criterion.
\end{abstract}

Keywords: Active vibration control, Journal hydrodynamic bearing, Motion stability, Lubrication stability, Movable bushing.

\section{Introduction}

The operating range of the radial journal bearings reduces the instability of the oil whirl type. After crossing some speed limit, the rotor starts to vibrate. Many theoretical and experimental works deal with the analysis of the instability of the plain bearings. Pioneering is a study done by Muszynska, who designed a model of the journal movement in the hydrodynamic sliding bearing (Muszynska, 1986). The validity of the model is confirmed by many publications based on solving the Reynolds equation.

As the first in this paper, the results obtained with the use of the test rig for the development of actively controlled sliding bearings. The motion of the journal in the fixed bushing during the run-up of the rotational speed at the constant rate is shown in Fig. 1. Journal rotation starts with a thin oil film between the moving surfaces. The movement of the journal seems chaotic, and the journal moves in the proximity of the bushing wall. Lubrication is referred to as boundary, i.e., unstable. Before starting the run-up, the motor did not rotate for a relatively long time. If the journal rotates before starting at low speed, there is a journal motion without oscillation. Boundary lubrication occurs if the bearing characteristic (called Stribeck number) $\mu N / P$ is greater than the limit value $3.8 \times 10^{-5}(\mathrm{~Pa} . \mathrm{s} \times \mathrm{rev} / \mathrm{s} / \mathrm{Pa})$ (Budynas, 2011). In the formula for the calculation of the bearing characteristic $\mu$ is an oil dynamic viscosity in Pa.s, $N$ a rotational speed of the rotor in rev/s and $P$ is a load per unit of projected bearing area in $\mathrm{N} / \mathrm{m}^{2}$ or Pa. The limit revolutions for boundary lubrication were $1200 \mathrm{rpm}$ at our test rig. Lubrication is considered to be stable with a thick oil film up to a speed of more than $1200 \mathrm{rpm}$ as shown in the diagrams in Fig. 1. However, the bearing pin movement is stable only up to about up $5000 \mathrm{rpm}$. When these speeds are exceeded, the axle of the bearing journal starts to circulate, causing the rotor to vibrate. These vibrations are called whirl. Figure 1 also shows running spectra that demonstrate the frequency spectrum of the motion of the bearing journal. The rotational frequency of the journal axis about the bushing bore centre is the $\lambda$ - multiple of the rotational speed of the rotor or journal $\Omega$ in $\mathrm{rad} / \mathrm{s}$. The size of the dimensionless

Prof. Ing. Jiř́ Tůma, CSc., FEng.: VŠB Technická univerzita, VŠB - TU Ostrava, 17. listopadu 2172/15; 708 00, Ostrava; CZ, jiri.tuma@vsb.cz

** Prof. Ing. Antonín Víteček, CSc., FEng.: VŠB Technická univerzita, VŠB - TU Ostrava, 17. listopadu 2172/15; 708 00, Ostrava; CZ, antonin.vitecek@vsb.cz 
parameter $\lambda$ is within these limits $0.42<\lambda<0.48$. When we assume stable lubrication, it is possible to create a deterministic mathematical model, which will allow assessing the effect of an active slide bearing control.

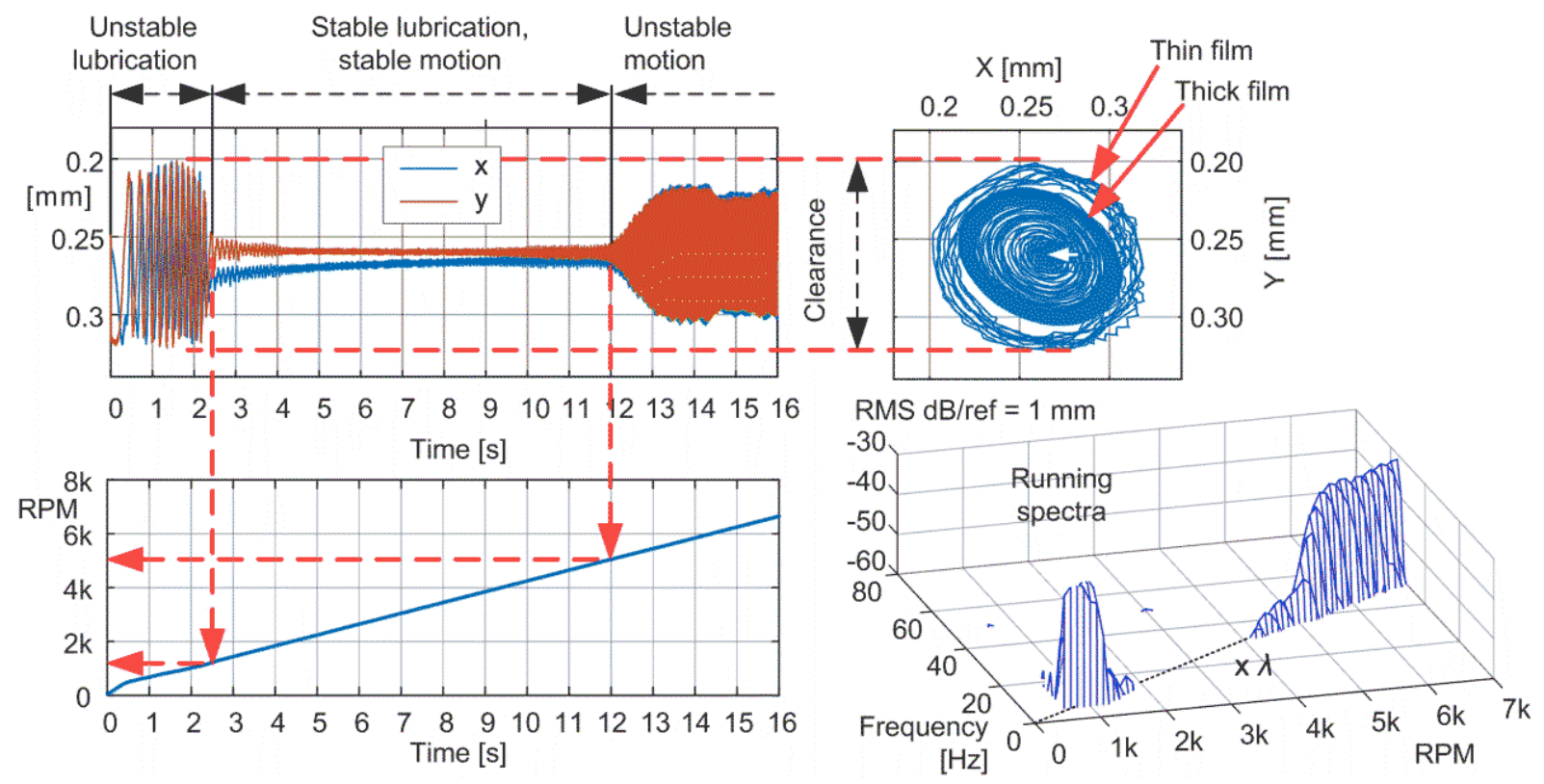

Fig. 1: The run-up of a journal bearing (Tưma et al., 2018).

\section{The mathematical model of the journal bearing with the fixed bushing}

The motion stability analysis at stable lubrication is focused on a single mass model with two degrees of freedom. The rotor is therefore supported by only one journal bearing, and the mass of the rotor moves in a plane perpendicular to the axis of rotation. The mathematical model of the linear time-invariant system is composed of two equations of motion, and the total system order is equal to four, which means that the system may be unstable. The stability analysis is focused on the effect of the rotor speed to determine the maximum operating speed of the rotor. This chapter deals with a conventional hydrodynamic journal bearing with a cylindrical bushing.

A)

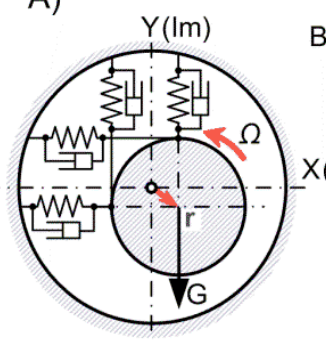

Model of the journal bearing

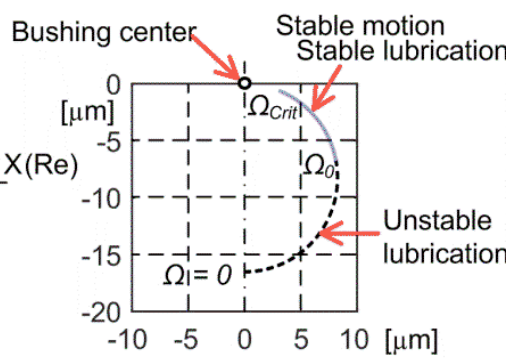

Trajectory of the journal axis

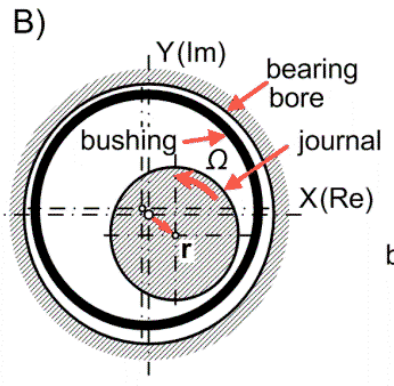

Coordinate system

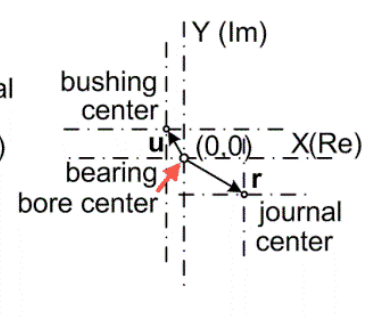

Fig. 2: 2 DoF model of a journal bearing.

A description of the bearing model is shown on the left of Fig. 2A. Coordinates are $x y$. Instead of Cartesian coordinates, a position vector $\boldsymbol{r}=x+j y$ in a complex plane can be used. The $x$ coordinate corresponds to the real axis and the $y$ imaginary axis. Motion equations designed by Muszynska. The same equations can be obtained by the analytical solution of the Reynolds equation (Tüma et al., 2018). Another confirmation of the suitability of the equation parameters selection is based on the numerical solution of the Reynolds equation published by Mendes and Cavalca (Mendes, et al. 2014). It concerns especially the dependence of stiffness parameter on the rotor rotational speed. The function type corresponds to the analytical solution. The motion equations are as follows

$$
\left[\begin{array}{cc}
M & 0 \\
0 & M
\end{array}\right]\left[\begin{array}{c}
\ddot{x} \\
\ddot{y}
\end{array}\right]+\left[\begin{array}{cc}
D & 0 \\
0 & D
\end{array}\right]\left[\begin{array}{c}
\dot{x} \\
\dot{y}
\end{array}\right]+\left[\begin{array}{cc}
K & D \lambda \Omega \\
-D \lambda \Omega & K
\end{array}\right]\left[\begin{array}{l}
x \\
y
\end{array}\right]=\left[\begin{array}{l}
F_{X} \\
F_{Y}
\end{array}\right]
$$


where $M$ is a mass of the rotor, $D$ is damping, $\lambda$ is the dimensionless fluid averaged circumferential velocity ratio and $\Omega$ is rotational speed of the rotor. We assume that the forces $F_{X}$ and $F_{Y}$ act on the bearing journal.

The block diagram corresponding to the system of equations (1) is shown on the left in Fig. 3. The block diagram on the right was a slight redraw of the diagram on the left side of Fig. 3 to obtain the closed loop arrangement. The transfer functions obtained using the Laplace transform are presented inside each block.
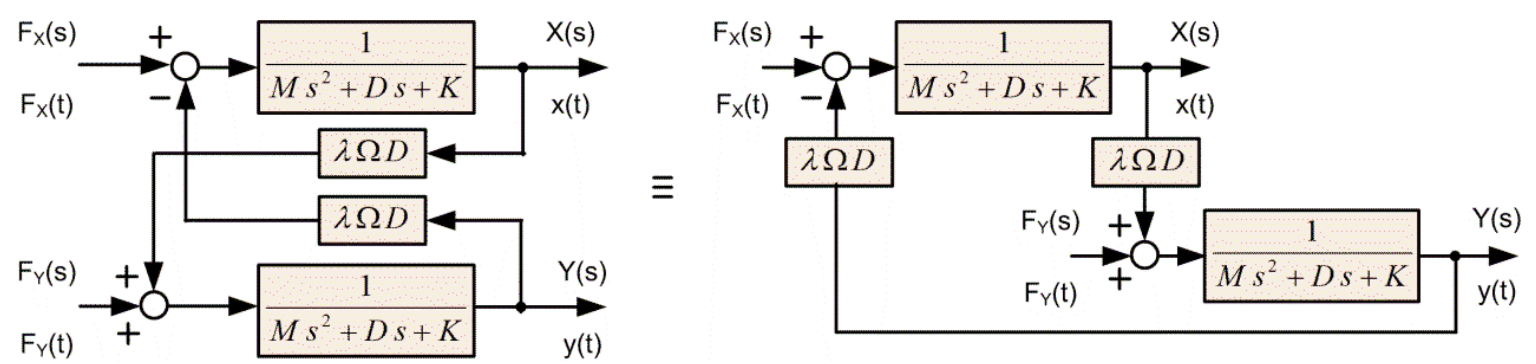

Fig. 3: The block diagram corresponding to the system of equations (1).

System stability will be analyzed using the Nyquist stability criterion (Tůma et al., 2013). The frequency transfer function of the open loop system $(s=j \omega)$ is the basis for a system stability verification

$$
\left[\frac{X(s)}{F_{X}(s)}\right]_{O L}=\frac{(D \lambda \Omega)^{2}}{\left(M s^{2}+D S+K\right)^{2}},\left[\frac{X(j \omega)}{F_{X}(j \omega)}\right]_{O L}=\frac{(D \lambda \Omega)^{2}}{\left(j D \omega+K-M \omega^{2}\right)^{2}}=\frac{(D \lambda \Omega)^{2}}{2 j D \omega\left(K-M \omega^{2}\right)+\left(K-M \omega^{2}\right)^{2}-D^{2} \omega^{2}} .
$$

If the gain of the open loop is equal to $-1+j 0$ then the closed-loop system is marginally stable or at the stability boundary and can oscillate with an angular frequency, which is called critical $\omega_{\text {Crit }}$, without any excitation. The critical frequency is calculated from a condition that the frequency transfer function is not a complex number but it is equal to a real value. The frequency transfer function (2) is a real number if $\omega=0$ or $K=M \omega^{2}$. The angular frequency resulting from the equation $K=M \omega^{2}$ is as follows $\omega=\sqrt{K / M}$. The transfer function of the open loop for the frequency $\sqrt{K / M}$ is equal to $-(D \lambda \Omega)^{2} /(D \omega)^{2}$. The critical frequency is equal to $\omega_{\text {crit }}=\lambda \Omega$. The rotor rotational speed for the system at the stability boundary is as follows

$$
\Omega_{\text {Crit }}=\omega_{\text {Crit }} / \lambda=\sqrt{K / M} / \lambda .
$$

For $\omega=0$, the journal axis trajectory under the gravity force $G$ which acts on the rotor is shown on the right in Fig. 2A. On the journal axis trajectory, besides $\Omega_{\text {Crit }}$, the rotational speed $\Omega_{0}=2 \pi N=$ $(2 \pi P / \mu) \times 3.8 \times 10^{-5}$ limiting the lubrication stability is also indicated.

\section{The mathematical model of the journal bearing with the non-rotating loose bushing}

The previous model assumed the existence of forces acting on the bearing journal. This operation is possible only for magnetic bearings. Our bearing is lubricated with an oil film, which is rotated to create an oil wedge lifting the journal. A movable bushing can only govern the movement of the journal. The external input for active control becomes the position of the bushing. First, we put position coordinates. The best way for the coordinate system is the use of positional vectors in a complex plane as is shown in Fig. 2B. The reference position is the center of the bushing bore or the position of the bearing body. The position of the journal axis indicates a position vector $\boldsymbol{r}=x+j y$, and the position of the axis of the bushing is labeled by $\boldsymbol{u}=u_{x}+j u_{y}$. The equation of motion according to Muszynska's model (1) with the use of complex variables

$$
M \ddot{\boldsymbol{r}}+D \dot{\boldsymbol{r}}+(K-j D \lambda \Omega) \boldsymbol{r}=\boldsymbol{F} .
$$

Substitution $\boldsymbol{r}-\boldsymbol{u}$ instead of $\boldsymbol{r}$ for force proportional to $\boldsymbol{r}$ and $\boldsymbol{r}$ introduces the control variable $\boldsymbol{u}$, which is a position of the bearing bushing. The bushing position affects only damping and stiffness of the oil film. The inertia force acting on the rotor depends only on $\ddot{\boldsymbol{r}}$. The force $\boldsymbol{F}$ is omitted because it is replaced by fluid forces exited by the oil film

$$
M \ddot{\boldsymbol{r}}+D \dot{\boldsymbol{r}}+(K-j D \lambda \Omega) \boldsymbol{r}=D \dot{\boldsymbol{u}}+(K-j D \lambda \Omega) \boldsymbol{u} .
$$

The block diagram representing equation (5) is shown on the left in Fig. 4. In this figure, on the right up, it is a simplified block diagram that contains complex quantities as inputs and outputs. A block diagram 
of a closed loop that contains a proportional controller is shown in this figure at the bottom on the right. The controller proportional gain is designated by $K_{P}$. The Laplace transfer function and frequency transfer function relating the position of the bushing and journal are as follows

$$
\frac{\boldsymbol{r}(s)}{\boldsymbol{u}(s)}=\frac{D s+(K-j D \lambda \Omega)}{M s^{2}+D s+(K-j D \lambda \Omega)}, \quad \frac{\boldsymbol{r}(j \omega)}{\boldsymbol{u}(j \omega)}=\frac{j D \omega+(K-j D \lambda \Omega)}{j D \omega+\left(K-j D \lambda \Omega-M \omega^{2}\right)} .
$$

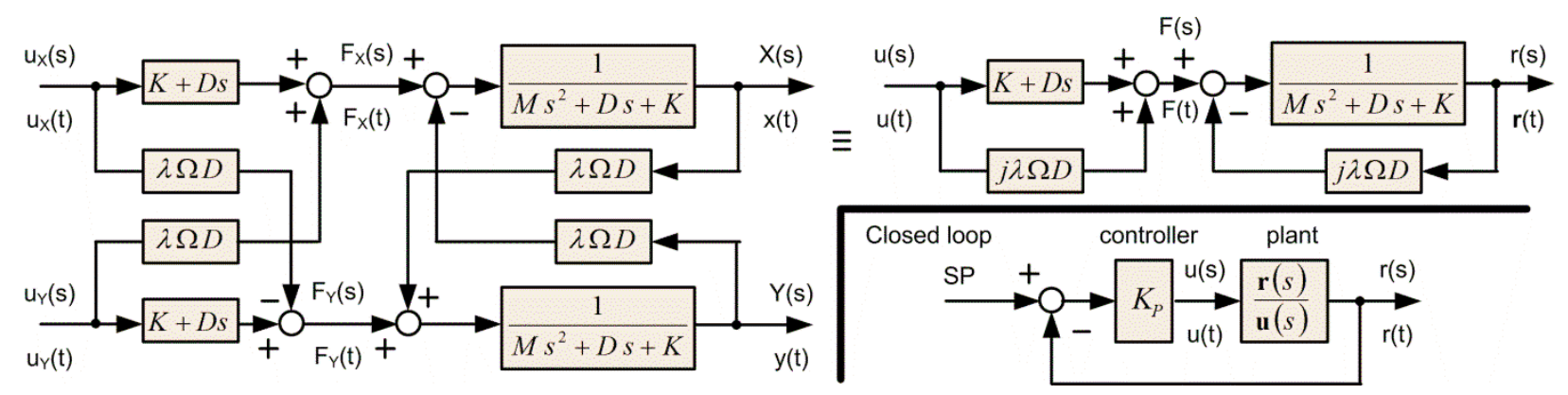

Fig. 4: The block diagram of the plain bearing with the non-rotating loose bushing.

The magnitude of the transfer function corresponding to the open loop which is equal to $-1+j 0$ determines the critical frequency $\omega_{\operatorname{Max}}$ of the closed loop system at the stability boundary

$$
K_{P} \frac{j D \omega+(K-j D \lambda \Omega)}{j D \omega+\left(K-j D \lambda \Omega-M \omega^{2}\right)}=-1+j 0 .
$$

The calculation procedure is the same as for formula (3). The left side of formula (6) is a real number if $\omega=0$ or $\omega=\lambda \Omega$. The critical rotor rotational speed at the stability boundary is as follows

$$
\Omega_{\text {Max }}=\sqrt{\left(K\left(1+K_{P}\right) / M\right)} / \lambda=\omega_{\text {Crit }} \sqrt{1+K_{P}} .
$$

The stiffness of the oil film has increased by the effect of the feedback, and it is $\sqrt{1+K_{P}}$-times greater than the film stiffness without the feedback, the limit angular velocity for stable operation of the journal bearing is also increased.

\section{Conclusions}

Analysis of the motion stability of the journal in the bearing bushing showed that feedback from the journal position relative to the bearing body increases the stable operational speed range proportionally to the square root of the proportional controller gain.

\section{Acknowledgment}

This research was supported by the European Regional Development Fund in the Research Centre of Advanced Mechatronic Systems project No. CZ.02.1.01/ 0.0/0.0/ 16_019/0000867 within the Operational Programme Research, Development and Education and also by the SGS project No. SP2019/51 Applied Research in the Area of Machine and Process Control.

\section{References}

Muszynska, A.(1986). Whirl and whip - rotor/bearing stability problems. Journal of Sound and Vibration. 1986, Vol. 110, Issue 3, (443-462).

Budynas, R.G. and Nisbett, J.K. (2011) Shigley's mechanical engineering design, 9th edition. New York: McGrawHill, 2011. ISBN 978-0-07-352928-8.

Mendes, R. U., and Cavalca, K. J. (2014) On the Instability Threshold of Journal Bearing Supported Rotors. International Journal of Rotating Machinery. Vol. 2014, Article ID 351261.

Tůma, J., Pawlenka, M., Mahdal, M. Kozubková, M. and Šimek, J. (2018) Theoretical and experimental analysis of the bearing journal motion due to the fluid force caused by the oil film. MM science journal, 2018, October.

Tuma, J., Simek, J., Skuta, J., and Los, J. (2013) Active vibrations control of journal bearings with the use of piezo actuators. Mechanical Systems and Signal Processing, 2013, Vol. 36, (618-629). 